Research Article

\title{
A putative RA-like region in the brain of the scale-backed antbird, Willisornis poecilinotus (Furnariides, Suboscines, Passeriformes, Thamnophilidae)
}

\author{
Jamily L.R. de Lima ${ }^{1}$, Fabricio A. Soares ${ }^{1}$, Ana C.S. Remedios ${ }^{1}$, Gregory Thom ${ }^{2}$, Morgan Wirthlin ${ }^{3}$, \\ Alexandre Aleixo ${ }^{2}$, Maria Paula C. Schneider ${ }^{1}$, Claudio V. Mello ${ }^{3}$ and Patricia N. Schneider ${ }^{1}$ \\ ${ }^{1}$ Instituto de Ciências Biológicas, Universidade Federal do Pará, Belém, PA, Brazil. \\ ${ }^{2}$ Museu Paraense Emilio Goeldi, Belém, PA, Brazil. \\ ${ }^{3}$ Department of Behavioral Neuroscience, Oregon Health \& Science University, Portland, OR, USA.
}

\begin{abstract}
The memorization and production of song in songbirds share important parallels with the process of speech acquisition in humans. In songbirds, these processes are dependent on a group of specialized telencephalic nuclei known as the song system: HVC (used as a proper name), RA (robust nucleus of arcopallium), LMAN (lateral magnocellular nucleus of the nidopallium) and striatal Area X. A recent study suggested that the arcopallium of the Sayornis phoebe, a non vocal learner suboscine species, contains a nucleus with some properties similar to those of songbird RA, suggesting that the song system may have been present in the last common ancestor of these groups. Here we report morphological and gene expression evidence that a region with some properties similar to RA is present in another suboscine, the Amazonian endemic Willisornis poecilinotus. Specifically, a discrete domain with a distinct Nissl staining pattern and that expresses the RA marker RGS4 was found in the arcopallium where the oscine RA is localized. Our findings, combined with the previous report on the $S$. phoebe, suggest that an arcopallial region with some RA-like properties was present in the ancestor of both Suboscines infraorders Tyranni and Furnarii, and is possibly an ancestral feature of Passeriformes.
\end{abstract}

Keywords: vocal learning, RGS4, song nuclei, in situ hybridization.

Received: January 29, 2015; Accepted: April 21, 2015.

\section{Introduction}

Vocal learning is a rare trait present in three clades of birds: hummingbirds (Trochiliformes), parrots (Psitaciformes) and songbirds (Oscines, Passeriformes) (Zigmond et al., 1973, Jarvis et al., 2000, Wilbrecht and Nottebohm, 2003, Arriaga et al., 2012). The memorization and production of song in avian species that learn their vocalizations share many important features with human speech (Doupe and Kuhl, 1999) and rely on a set of discrete telencephalic nuclei referred to as the song control system (Wilbrecht and Nottebohm, 2003, Jarvis, 2004, Arriaga et al., 2012). Recent phylogenetic data have solidified the idea that parrots and songbirds are sister taxa, while hummingbirds are a separate group (Jarvis et al., 2014) thus indicating that vocal learning, which is absent in the intervening clades, most likely evolved convergently among avian vocal learners (Jarvis, 2004).

Vocal control in avian vocal learners is dependent upon two main pathways. The posterior pathway is composed of HVC (used as a proper name) and RA (robustus

Send correspondence to Patricia N. Schneider. Instituto de Ciências Biológicas, Universidade Federal do Pará, 66055170 Belém, Pará, Brazil. E-mail: schneider@ufpa.br. nucleus of the arcopallium), which are localized in the caudal part of the telencephalon and are involved in the motor encoding of learned vocalizations. RA provides the major output of the song system; it influences vocal production through direct and indirect projections to brainstem motor neurons that innervate the syrinx, the vocal organ of birds. The anterior pathway resembles mammalian cortico-basal ganglia-thalamo cortical loops, and is more directly involved in vocal learning and plasticity. It includes two nuclei located rostrally in the telencephalon: Area X of the striatum (Area $\mathrm{X}$ ) and the lateral magnocellular nucleus of the anterior nidopallium (LMAN) (Jarvis, 2004, Prather, 2013) (Figure 1A).

The Passeriformes are composed of two suborders: oscines (songbirds), with the largest number of species, and suboscines. The latter are further divided into two main infraorders: Tyranni and Furnarii. Representatives of the Tyranni are the tyrant-flycatchers, cotingas and phoebes, whereas antbirds are the most numerous species within the Furnarii infraorder. While traditionally viewed as vocal non-learners (Brenowitz et al., 1991, Touchton et al., 2014), there is also evidence that some suboscines, espe- 


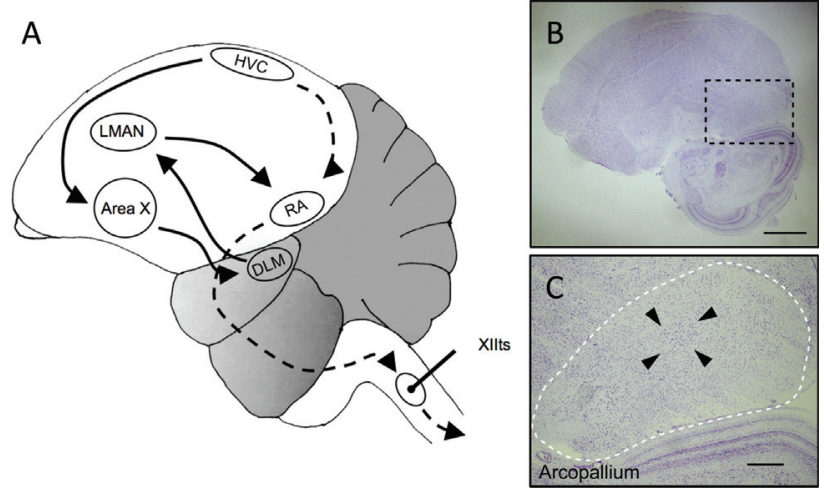

Figure 1 - The song control system of the Pale-breasted Thrush, Turdus leucomelas. (A) Schematic representation of the song control system in an oscine brain, showing the song production pathway (dashed arrows) and the song learning pathway (arrows) (Adapted from Nottebohm, 2005). (B) Nissl stained brain section of T. leucomelas, the arcopallium is located within the dashed line box. (C) The archopallium is demarcated by white dashed line, with arrowheads indicating the location of the RA nucleus. Scale bars are $20 \mu \mathrm{m}(\mathrm{B})$ and $5 \mu \mathrm{m}$ (C).

cially within the Cotingidae, may present at least some rudimentary forms of vocal learning (Irestedt et al., 2002).

Early sudies utilizing cytoarchitectonics criteria in Nissl-stained sections suggested that suboscines lack the song system nuclei found in oscines (Brenowitz et al., 1991). A recent study, however, provided evidence that the Eastern phoebe (Sayornis phoebe), a tyrant flycatcher, has a rudimentary circuitry with a nucleus that shares some properties of the oscine RA, and that lesions to this region leads to vocal impairements similar to those observed with RA lesions (Liu et al., 2013). Importantly, however, when their juveniles are raised in isolation, without exposure to tutor song, they develop normal conspecific adult vocalizations, indicating lack of vocal learning in this species (Brenowitz et al., 1991). Similar results have been obtained with an antbird (Hylophylax naevidoides), providing support for a lack of vocal learning, among suboscines (Touchton et al., 2014). Due to these conflicting findings, the extent to which vocal learning and associated brain structures are prevalent among suboscines remains unsettled.

In this study, we searched for the presence of song nuclei circuitry in yet another suboscines species, the antbird Willisornis poecilinotus, from an infraorder that has not yet been examined with regards to this trait. We found evidence of the presence of a rostro-ventral arcopallial area with some cytoarchitetonic features resembling those of song nucleus RA. Furthermore, we found evidence that RGS4, a marker of song nucleus RA in songbirds, is selectively expressed within this nucleus. These data, together with previous reports on Sayornis phoebe, suggests that some possible components of the circuitry associated with the emergence of vocal learning could be ancestral to Passeriformes.

\section{Material and Methods}

\section{Sampling}

A total of 6 male specimens of Willisornis poecilinotus and 2 male specimens of Turdus leucomelas were captured at the Refúgio de Vida Silvestre Metrópole da Amazônia, in Belém. Brains were dissected out and flash frozen in Tissue-tek embedding medium on dry ice and $100 \%$ ethanol. Cryosections $(10 \mu \mathrm{m})$ were made using a Leica CM1850 UV cryostat and placed onto colorfrost plus microscope slides, fixed in 3\% paraformaldehyde, and stored at $-80^{\circ} \mathrm{C}$ for further use. This study was approved by the Ethics Committe at the Instituto de Ciências Biológicas and performed in accordance to the special licence 20902-1 issued to MPDS by IBAMA/SISBIO.

\section{Riboprobe synthesis}

The cDNA clones for parvalbumin (PVALB) and for the regulator of G-protein signaling 4 (RGS4) were from the zebra finch brain cDNA ESTIMA collection, cloned in pbluescript II KS plasmids. As previously performed for the zebra finch, the specificity of these probes was verified by performing a blat alignment (using the UCSC genome browser) to the genome sequence of the golden-collared manakin (Manacus vitellinus), a subsocine species; the analysis showed alignment to the correct ortholog and lack of significant hits to other loci, supporting probe specificity in this avian suborder. Polymerase Chain Reaction (PCR) amplification was performed using forward and reverse primers (IDT) with a T3 overhang (Reverse: 5'-GTA AAA CGA CGG CCA GTG AG-3' and Forward: 5'-ATG ACC ATG ATT ACG CCA AG-3') to amplify the cDNA insert and $\mathrm{T} 3$ polymerase promoter using the vector as template. PCR was performed in $50 \mu \mathrm{L}$ reaction volumes containing 39.2 $\mu \mathrm{L}$ of RNase free water, $1.5 \mu \mathrm{L}$ of $10 \mathrm{mM} \mathrm{MgCL}_{2}$, $5 \mu \mathrm{L}$ of $10 \mathrm{x}$ buffer, $1 \mu \mathrm{L}$ of each primer $(0.5 \mu \mathrm{M}), 1 \mu \mathrm{L}$ of dNTP mix $(10 \mathrm{mM}), 0.3 \mu \mathrm{L}$ of Taq DNA Polymerase, and $1 \mu \mathrm{L}$ of DNA template. The temperature profile consisted of preheating at $94{ }^{\circ} \mathrm{C}$ for $3 \mathrm{~min}, 32$ cycles of denaturation at $94{ }^{\circ} \mathrm{C}$ for $45 \mathrm{~s}$, annealing at $55^{\circ} \mathrm{C}$ for $30 \mathrm{~s}$, and extension at $72{ }^{\circ} \mathrm{C}$ for $90 \mathrm{~s}$, followed by a final extension step at $72{ }^{\circ} \mathrm{C}$ for $10 \mathrm{~min}$. Then, antisense riboprobes were synthesized using T3 RNA polymerase (Life Technologies) and DIG-labeling mix (Roche). The riboprobe reaction (Life Technologies) was performed in $20 \mu \mathrm{L}$ reaction volumes containing $0.5 \mu \mathrm{L}$ of RNAse inhibitor, $2 \mu \mathrm{L}$ of DTT, $2 \mu \mathrm{L}$ of DIG, $2 \mu \mathrm{L}$ of $10 \mathrm{x}$ reaction buffer, $5 \mu \mathrm{L}$ of template, $2 \mu \mathrm{L}$ of T3 enzyme mix, and $6.5 \mu \mathrm{L}$ of nuclease-free water.

\section{Nissl Staining}

Cryosections were stained in an aqueous solution of $0.1 \%$ cresyl violet. After staining, the slides were dehydrated in absolute ethanol for $2 \mathrm{~min}$; and cleared with 
xylene for 2 min twice. The sections were then coverslipped using CytosealXYL (Richard-Allan Scientific) mounting medium.

\section{In situ hybridization}

In situ hybridization was performed according to a previously established protocol, with modifications (Lovell et al., 2013, Carleton et al., 2014). Brain sections were acetylated for $10 \mathrm{~min}$ in a solution of $0.33 \%$ acetic anhydride in water, rinsed twice with $2 x$ SSPE buffer (0.02 M EDTA and $2.98 \mathrm{M} \mathrm{NaCl}$ in $0.2 \mathrm{M}$ phosphate buffer) and dehydrated in $70 \%, 95 \%$ and $100 \%$ RNA-free ethanol. Each section was then hybridized with a solution $(16 \mu \mathrm{L})$ containing $50 \%$ formamide, $2 x$ SSPE, $1 \mu \mathrm{g} / \mu \mathrm{l} \mathrm{BSA}, 1 \mu \mathrm{g} / \mu \mathrm{L}$ poly-A (New England Biolabs) in DEPC-treated water, and $1 \mu \mathrm{L}$ of the DIG-labeled riboprobe. Slides were coverslipped, sealed by immersion in mineral oil, and incubated overnight at $65^{\circ} \mathrm{C}$. On the following day, sections were rinsed and de-coverslipped in chloroform, washed briefly in $2 \mathrm{x}$ SSPE, and incubated serially for $1 \mathrm{~h}$ and $10 \mathrm{~min}$ at $65^{\circ} \mathrm{C}$ in 2x SSPE containing $50 \%$ formamide, and twice in $0.1 \mathrm{x}$ SSPE for $30 \mathrm{~min}$ at $65^{\circ} \mathrm{C}$. Sections were then rinsed briefly in TNT (TN: $100 \mathrm{mM}$ Tris, pH 7.5; $150 \mathrm{mM} \mathrm{NaCl} / \mathrm{TNT}$ : $\mathrm{TN}+0.3 \%$ Triton-X 100), demarcated using a pap pen (Life Technologies) and blocked for $30 \mathrm{~min}$ at RT in $200 \mu \mathrm{L}$ of TNB $(0.1 \mathrm{M}$ Tris, $150 \mathrm{mM} \mathrm{NaCl})$ blocking buffer (200 $\mu \mathrm{L} \mathrm{TNB/slide,} \mathrm{1 \%} \mathrm{skim} \mathrm{milk)} \mathrm{and} \mathrm{incubated} \mathrm{over-}$ night in TNB with an alkaline phosphatase conjugated anti-DIG antibody (Roche) (TNB $+0,3 \%$ skim milk + anti-DIG-AP; 1:600 dil.). Then, the slides were washed twice for $15 \mathrm{~min}$ in TMN (100 mM Tris, $\mathrm{pH} 9.5 ; 150 \mathrm{mM}$ $\mathrm{NaCl}, 0.05 \mathrm{M} \mathrm{MgCl}$ ), and incubated for $1-3$ days in a detection solution containing the alcaline phosphatase substrates Nitro-Blue Tetrazolium Chloride (NBT)(Roche) and 5Bromo-4-Chloro-3-Indolyl-phosphate p-Toluidine Salt (BCIP) (Roche) in a TMN solution with 1:1 chromogenic proportion. Slides were washed overnight in distilled water to remove salts, fixed in $4 \%$ paraformaldehyde, washed twice in distilled water, and coverslipped with Aquamount (Fischer Scientific). Slides were imaged using a Nikon SMZ1500 microscope and processed on NIS-Elements D 4.10.01 program (Nikon). For qualitative assessment of mRNA expression in brain tissue we compared the neuroanatomical expression of these two selected genes with data retrieved from the ZEBrA database (www.zebrafinchatlas.org).

\section{Results}

\section{Cytoarchitectural analysis of the arcopallium of W. poecilinotus in Nissl-stained brain sections}

As previosuly described in several songbird species, the set of song nuclei that controls vocal learning displays a characteristic cytoachitetonic organization and conserved locations within the forebrain. To establish whether similar nuclei are also present in the antbird $W$. poecilinotus, we first examined Nissl-stained parasagital brain sections of adult male Pale-breasted Thrush, Turdus leucomelas (Turdidae), a vocal-learning oscine species, thereby establishing a basis for comparison in a similar-sized oscine brain. The neuronal cells present in the song nuclei of this species are larger and noticeably distinct from the surrounding areas (Figure 1B,C). To characterize the forebrain of $W$. poecilinotus, we studied the general organization of the brain utilizing Nissl-stained parasagittal sections from adult males. After a thorough analysis, discrete nuclei with the large cells characteristic of HVC, LMAN and Area X in vocal learners were not observed in any of the Nissl-stained sections (Figure 2A,C,E). However, we found that the arcopallium contained discrete nuclei with large neuronal

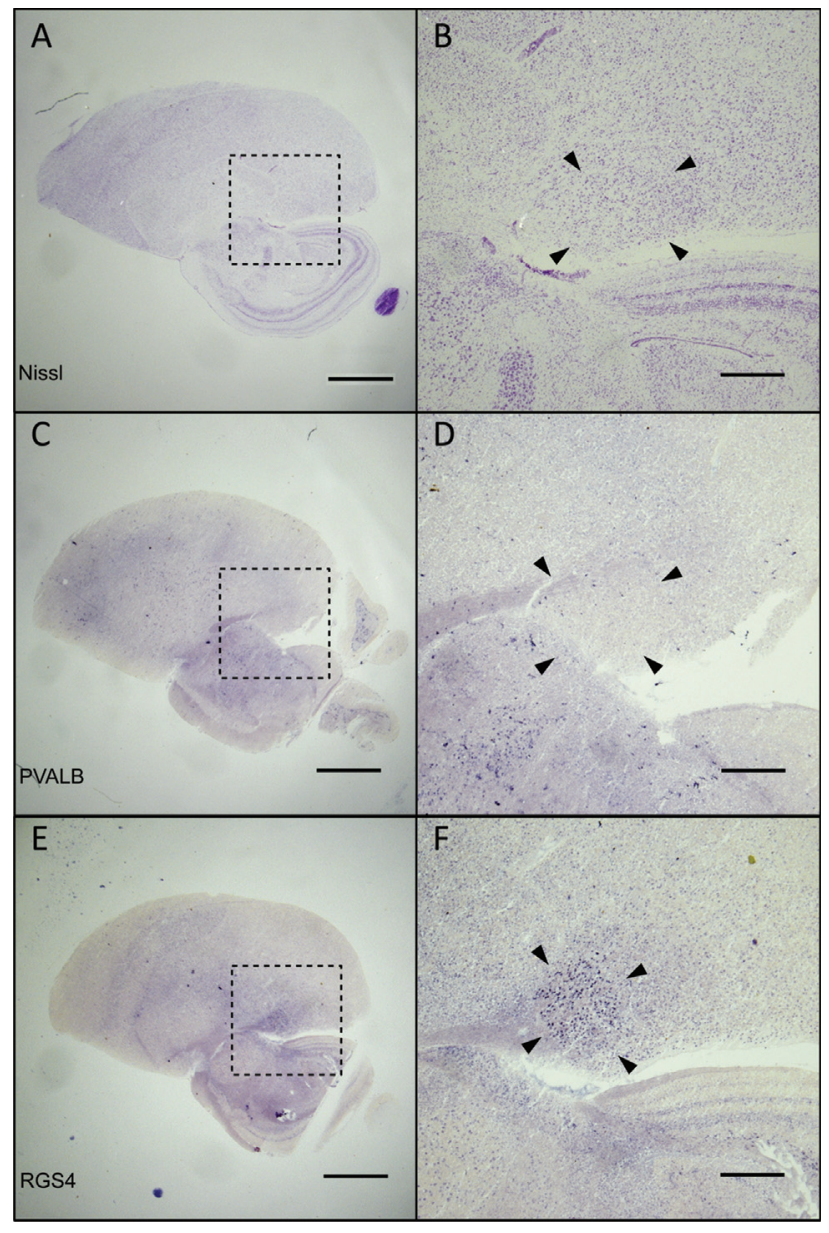

Figure 2 - An RA-like region in the brain of the antbird Wlisornis poecilinotus. (A) Nissl stained section of the $W$. poecilinotus brain, with the arcopallium region denoted by a dashed line box. (B) RA like nucleus characterized by the presence of large neuronal cells that are distinct from the surrounding cells. (C) Expression pattern of the song nuclei marker PVALB in a brain section of $W$. poecilinotus and (D) within the arcopallium. (E) Expression pattern of the song nuclei marker RGS4 in a brain section of $W$. poecilinotus and (F) within the arcopallium. Arrowheads indicate the location of the RA-like nucleus. Scale bars are $20 \mu \mathrm{m}$ (A, C, E) and $5 \mu \mathrm{m}(\mathrm{B}, \mathrm{D}, \mathrm{F})$. 
cells resembling those of song nuclei in oscines, in particular a prominent one within the rostro-ventral region (Figure $2 \mathrm{~A}, \mathrm{~B}$ and Figure $\mathrm{S} 1$ ); this prompted us to further examine a putative RA-like nucleus utilizing molecular criteria.

\section{Expression pattern of oscine song nuclei markers in $W$. poecilinotus}

To further investigate the presence of song nuclei in $W$. poecilinotus, the expression pattern of PVALB and RGS4 were analyzed. These markers were chosen as they represent conserved markers of song nuclei, including RA, in songbirds (Lovell et al., 2008) and ZEBrA database (www.zebrafinchatlas.org) and in other avian vocal learners (Hara et al., 2012). Similar to our Nissl staining results, neither PVALB nor RGS4 expression was detected in putative HVC, LMAN and Area X nuclei (Figure 2C-E). Although PVALB expression was not detected in the arcopallium or in any putative song control nucleus (Figure 2C and D), it was observed in the cerebellum (Figure S2), indicating at least partial probe cross-hybridization. Nevertheless, we found enriched RGS4 mRNA in the arcopallium (Figure 2E,F and Figure S3), within a rostro-ventral region that overlapped with the location of the distinct cytoarchitectonic region detected by Nissl staining.

\section{Discussion}

Our data provide evidence for the presence of a distinct region within the arcopallium of $W$. poecilinotus, a suboscine representative, with some cytoarchitectonic and molecular similarities with the RA song nucleus of songbirds and its analogous nuclei in hummingbirds and parrots. The cytoarchitetonic analysis of Nissl-stained brain sections revealed distinct large cells within a discrete domain in the arcopallium, as characteristic of RA and other song nuclei in songbirds. To further examine the presence of areas with song-nuclei properties in a suboscine species, we analized the expression of two song nuclei markers. RGS4 is a regulator of $\mathrm{G}$ protein signaling that controls the expression of NMDA-receptors in the forebrain on song learners (Saugstad et al., 1998). This gene has androgenrelated action and its expression is highly enriched in the RA nucleus of songbirds (Saugstad et al., 1998, Wild et al., 2005, Lovell et al., 2008). Our results showed enriched mRNA expression of this song nuclei marker within the arcopallium of $W$. poecilinotus, suggesting the presence of a potential RA-like region in a suboscine representative. However, no differential expression was observed in HVC, Area $\mathrm{X}$ or in the LMAN putative areas in this species.

One possible interpretation is that the arcopallium of $W$. poecilinotus contains a nucleus analogous to oscine RA that is possibly part of a vocal control circuitry and has some involvement in vocal control. While connectivity and functional data in this species are still lacking, such an in- terpretation would be consistent with that suggested by recent data obtained in the Eastern phoebe, a tyrant flycatcher suboscine. That study used gene expression analysis to demonstrate singing related activation of a discrete arcopallial domain, and lesions to demonstrate that some vocal changes occur after the loss of their so-called RA-like region (Liu et al., 2013). Taken together, these studies suggest that an arcopallial region with some similarities to song nucleus RA and thus possibly representing a rudimentary form of RA is present in suboscines species from both tyranni and furnarii infraorders (Figure 3 ). Such a nucleus could have been present in the common ancestor to oscines and suboscines and thus possibly be ancestral to Passeriformes.

In vocal learning birds, RA and its analogs in parrots and hummingbirds are the main output of the circuitry involved in learned vocalizations, and are thought to play central roles in controlling learned songs (Patton et al., 1981, Nottebohm et al., 1982). Namely, they receive projections from HVC and LMAN and project to motorneurons that innervate the syrinx, the main avian vocal organ (Vates and Nottebohm, 1995). The neuronal activation patterns in RA during singing correlate with specific features of song (Yu and Margoliash, 1996, Hahnloser et al., 2002) indicating that it plays an important role in the neuronal encoding of learned song patterns. The role that an RA-like region might play in suboscines is not understood, especially in species where there is no compelling evidence of vocal learning. Also, we did not observe the same expression pattern using the song nuclei marker PVALB, indicating that, the RA-like region, represents the rudimentary form of RA. By analogy to songbirds, one would expect such a region to project to vocal and respiratory motorneurons in the brainstem, and to be functioanally active during vocal behaviors. While such evidence is still lack-

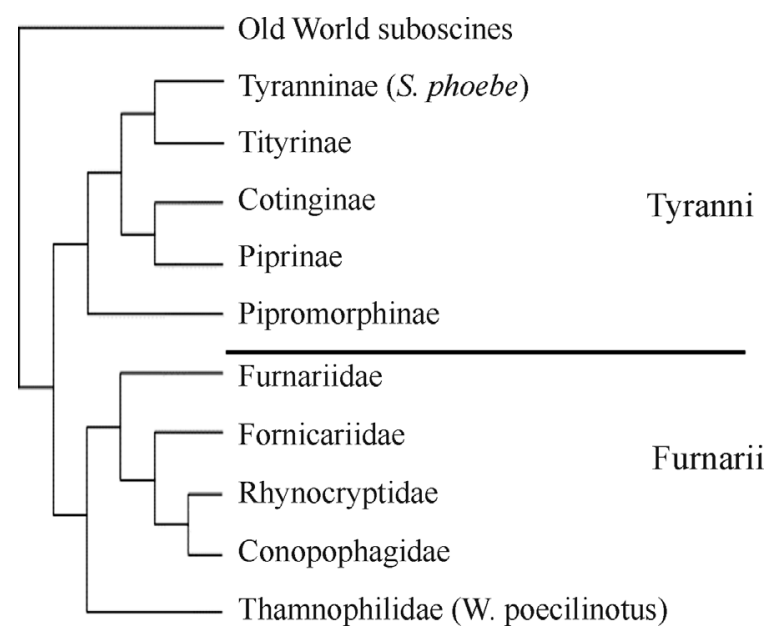

Figure 3 - Phylogenetic tree of the suborder suboscines. New world suboscines are divided into the infraorders Tyranni and Furnarii. Putative RA-like nuclei have been found in S. phoebe (Liu et al., 2013) and in Willisornis poecilinotus (this study). 
ing, the area identified here is now an interesting candidate target for such studies.

Importantly, however, there are alternative interpretations to our findings. For example, the domain of enriched RGS4 expression is located rostro-ventrally, underneath the occipitomesencephalic $(\mathrm{OM})$ tract, thus not entirely consistent with the expected location of an RA-like nucleus based on songbirds. Rather, this domain is very reminiscent of a rostroventral arcopallial domain of high RGS4 expression that can also be seen in the zebra finch (ZEBrA atlas; www.zebrafinchatlas.org) and could subserve other, as yet undetermined function(s), such as the auditory processing circuit, part of the conserved auditory pathways, as it sends descending projections to the auditory thalamus and midbrain (Wild et al., 2005). Furthermore, some of the induced gene expression and lesion effects observed in the Eastern phoebe study could be interpreted by considering that the so-called RA-like nucleus may be part of a conserved auditory processing circuit (Wild et al., 2005), analogous to the region immediately outside of RA (also called the RA cup) in a songbird (Mello et al., 1998). That region shows singing-induced auditory activation (Mello and Clayton, 1994), but possible effects of lesions have not yet been examined in a songbird.

\section{Acknowledgments}

The authors thank MPEG (Museu Paraense Emilio Goeldi) for the access to the specimens used in this study and The Songbird Neurogenomics and Dr. Peter Lovell for providing the cDNA clones and helpful discussions and suggestions on the manuscript. Funding by the Brazilian Avian Genome Consortium (CNPq/FAPESPA-SISBIO Aves) is acknowledged.

\section{References}

Arriaga G, Zhou EP and Jarvis ED (2012) Of mice, birds, and men: The mouse ultrasonic song system has some features similar to humans and song-learning birds. PLoS One 7:e46610.

Brenowitz EA, Nalls B, Wingfield JC and Kroodsma DE (1991) Seasonal changes in avian song nuclei without seasonal changes in song repertoire. J Neurosci 11:1367-1374.

Carleton JB, Lovell PV, McHugh A, Marzulla T, Horback KL, Mello CV (2014) An optimized protocol for highthroughput in situ hybridization of zebra finch brain. Cold Spring Harb Protoc 2014:1249-1258.

Doupe AJ and Kuhl PK (1999) Birdsong and human speech: Common themes and mechanisms. Annu Rev Neurosci 22:567-631.

Hahnloser RH, Kozhevnikov AA and Fee MS (2002) An ultra-sparse code underlies the generation of neural sequences in a songbird. Nature 419:65-70.

Hara E, Rivas MV, Ward JM, Okanoya K and Jarvis ED (2012) Convergent differential regulation of parvalbumin in the brains of vocal learners. PLoS One 7:e29457.
Irestedt M, Fjeldsa J, Johansson US and Ericson PG (2002) Systematic relationships and biogeography of the tracheophone suboscines (Aves, Passeriformes). Mol Phylogenet Evol 23:499-512.

Jarvis ED (2004) Learned birdsong and the neurobiology of human language. Ann NY Acad Sci 1016:749-777.

Jarvis ED, Ribeiro S, da Silva ML, Ventura D, Vielliard J and Mello CV (2000) Behaviourally driven gene expression reveals song nuclei in hummingbird brain. Nature 406:628632.

Jarvis ED, Mirarab S, Aberer AJ, Li B, Houde P, Li C, Ho SY, Faircloth BC, Nabholz B, Howard JT, et al. (2014) Wholegenome analyses resolve early branches in the tree of life of modern birds. Science 346:1320-1331.

Liu WC, Wada K, Jarvis ED and Nottebohm F (2013) Rudimentary substrates for vocal learning in a suboscine. Nat Commun 4:e2082.

Lovell PV, Clayton DF, Replogle KL and Mello CV (2008) Birdsong "transcriptomics": Neurochemical specializations of the oscine song system. PLoS One 3:e3440.

Lovell PV, Carleton JB and Mello CV (2013) Genomics analysis of potassium channel genes in songbirds reveals molecular specializations of brain circuits for the maintenance and production of learned vocalizations. BMC Genomics 14:e470.

Mello CV and Clayton DF (1994) Song-induced ZENK gene expression in auditory pathways of songbird brain and its relation to the song control system. J Neurosci 14:6652-6666.

Mello CV, Vates GE, Okuhata S, and Nottebohm F (1998) Descending auditory pathways in the adult male zebra finch (Taeniopygia guttata). J Comp Neurol 395:137-160.

Nottebohm F (2005) The neural basis of birdsong. PLoS Biol 3:e164.

Nottebohm F, Kelley DB and Paton JA (1982) Connections of vocal control nuclei in the canary telencephalon. J Comp Neurol 207:344-357.

Patton JE, Routh DK and Offenbach SI (1981) Televised classroom events as distractors for reading-disabled children. J Abnorm Child Psychol 9:355-370.

Prather JF (2013) Auditory signal processing in communication: Perception and performance of vocal sounds. Hear Res 305:144-155.

Saugstad JA, Marino MJ, Folk JA, Hepler JR, and Conn PJ (1998) RGS4 inhibits signaling by group I metabotropic glutamate receptors. J Neurosci 18:905-913.

Touchton JM, Seddon N and Tobias JA (2014) Captive rearing experiments confirm song development without learning in a tracheophone suboscine bird. PLoS One 9:e95746.

Vates GE, and Nottebohm F (1995) Feedback circuitry within a song-learning pathway. Proc Natl Acad Sci USA 92:51395143.

Wilbrecht L and Nottebohm F (2003) Vocal learning in birds and humans. Ment Retard Dev Disabil Res Rev 9:135-148.

Wild JM, Williams MN, Howie GJ and Mooney R (2005) Calcium-binding proteins define interneurons in $\mathrm{HVC}$ of the zebra finch (Taeniopygia guttata). J Comp Neurol 483:76-90.

Yu AC and Margoliash D (1996) Temporal hierarchical control of singing in birds. Science 273:1871-1875.

Zigmond RE, Nottebohm F and Pfaff DW (1973) Androgenconcentrating cells in the midbrain of a songbird. Science 179:1005-1007. 


\section{Supplementary Material}

The following online material is available for this article:

Figure S1 - Nissl stained section of the $W$. poecilinotus brain.

Figure S2 - Expression pattern of PVALB in a brain section of $W$. poecilinotus.
Figure S3 - RA-like region in the brain of the antbird $W$. poecilinotus

This material is available as part of the online article from http://www.scielo.br/gmb.

Associate Editor: Igor Schneider

License information: This is an open-access article distributed under the terms of the Creative Commons Attribution License, which permits unrestricted use, distribution, and reproduction in any medium, provided the original work is properly cited. 